\title{
A versatile trigger and synchronization module with IEEE1588 capabilities and EPICS support
}

\author{
J.M. López ${ }^{\mathrm{a}, *}$, M. Ruiz ${ }^{\mathrm{a}}$, J. Borrego ${ }^{\mathrm{a}}$, G. de Arcas $^{\mathrm{a}}$, E. Barrera ${ }^{\mathrm{a}}$, J. Vega ${ }^{\mathrm{b}}$ \\ a Crapo de Imvestigación en instizmentación y Acüstica Aplicada, Universidad Politếcnica de Madrid, Crta. Valencia Kim-7, Madrid 28a3ı, Spain \\ b Asociacion EURATOM/CIEMAT para Fusion, Madrid, Spain
}

\section{A R T I C L E I N F O}

\section{Keywords:}

Data acquisition

EPICS

IEEE 1588

Real time data

Processing protocol time precision

\begin{abstract}
A B S T R A C T
Event timing and synchronization are two key aspects to improve in the implementation of distributed data acquisition ( $d D A Q$ ) systems such as the ones used in fusion experiments. It is also of great importance the integration of dDAQ in control and measurement networks. This paper analyzes the applicability of the IEEE1588 and EPICS standards to solve these problems, and presents a hardware module implementation based in both of them that allow adding these functionalities to any DAQ. The JEEE1588 standard facilitates the integration of event timing and synchronization mechanisms in distributed data acquisition systems based on JEEE 803.3 (Ethernet). An optimal implementation of such system requires the use of network interface devices which include specific hardware resources devoted to the lEE 1588 functionalities. Unfortunately, this is not the approach followed in most of the large number of applications available nowadays. Therefore, most solutions are based in software and use standard hardware network interfaces. This paper presents the development of a hardware module (GI2E) with IEEE1588 capabilities which includes USB, RS232, RS485 and CAN interfaces. This permits to integrate any DAQ element that uses these interfaces in dDAQ systems in an efficient and simple way. The module has been developed with Motorola's Coldfire MCF5234 processor and National Semiconductors's PHY DP83640T, providing it with the possibility to implement the PTP protocol of [EEE1588 by hardware, and therefore increasing its performance over other implementations based in software. To facilitate the integration of the dDAQ system in control and measurement networks the module includes a basic Input/Output Controller (IOC) functionality of the Experimental Physics and Industrial Control System (EPICS) architecture. The paper discusses the implementation details of this module and presents its applications in advanced dDAQ applications in the fusion community.
\end{abstract}

\section{Introduction}

Synchronization is a key aspect of all distributed data acquisition systems, but is of special relevance in those systems used in fusion devices due to the wide range of diagnostics and requirements available. Different scenarios can be found, from the need of the typical single global trigger signal for short pulse fusion devices, to the need of several trigger signals for windowed acquisitions in long pulse devices, or the combination of both. The Precision Time Protocol (IEEE1588) presents a synchronization method for distributed systems that guarantees a high degree of synchronization between the clocks of the different elements of the system, and which permits to improve the trigger process of distributed systems. Precision Time Protocol (PTP) uses a multicasting procedure to send messages for synchronization from master to slaves devices on the network. Master and slaves interchange different messages in order to measure network delays and synchronize their clocks [1].

This paper presents the design of an electronic custom hardware which implements the PTP protocol for its use in distributed data acquisition systems, specifically in Experimental Physics and Industrial Control Systems (EPICS). EPICS is an architecture for developing scalable distributed systems with interesting characteristics from an implementation point of view, such as: open code, documentation, toolkits, etc. It is widely adopted in the scientific community $[2,9,10]$. The module designed includes a basic Input/Output Controller (IOC), and is based in a 32 bits microcontroller from Freescale's Coldfire family processors running RTEMS operating systems and has USB, RS232, RS485 and CAN interfaces. The availability of different interfaces permits to use this module with instruments that do not originally support this synchronization mechanism. Another advantage offered is the possibility to use instrumentation with reduced processing capabilities, or which does not support the EPICS protocols, such as standard multime- 


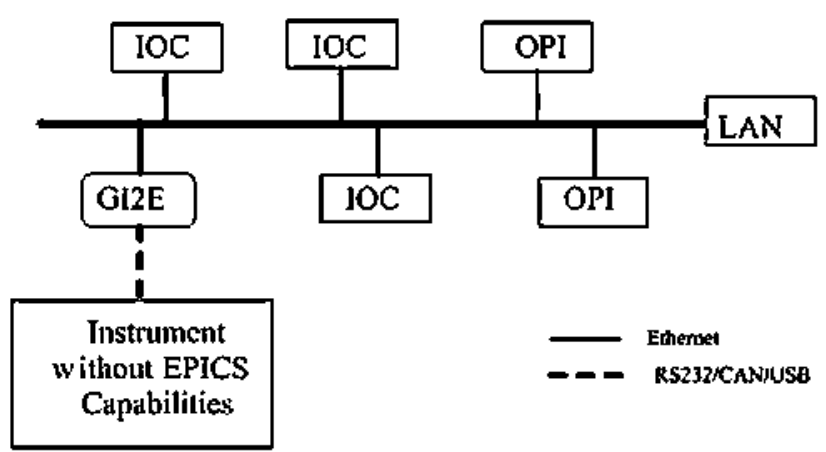

Fig. 1. EPICS data acquisition and control system using GI2E.

ters, PlCs, actuators and specific control elements in distributed DAQ systems.

The board has been developed by the Universidad Politécnica de Madrid and CIEMAT, and has been used in the Intelligent Test Measurement System (ITMS) platform to expand its synchronization mechanisms and to integrate standard measurement instrumentation.

\section{System architecture}

EPICS is an architecture that permits to implement distributed control system over local area networks (LAN). Its most relevant characteristic is that it is fully distributed, as every layer is independent of the underlying hardware. There is no need for central nodes, which facilitates scalability and robustness as there are no critical nodes. And system maintenance is also improved. An EPICS solution consists of the following elements:

- Operator Interface (OP) is a terminal that runs all tolls for data visualization and exploitations.

- Input/Output Controller (IOC) is usually a VME/VXI chassis containing one or more processors, input/output modules and any other standard interconnect bus such as GPIB.

- Local Area Network (LAN) is the physical media that supports communication between the different lOCs and OPIs of the system.

The system that has been developed, named Generic Instruments to EPICs (GI2E) module, is meant to be used to include any instrument that does not natively support EPICS in any EPICS based control applications through the use of standard interfaces such as USB, RS232, RS485 and CAN as shown in Fig. 1.

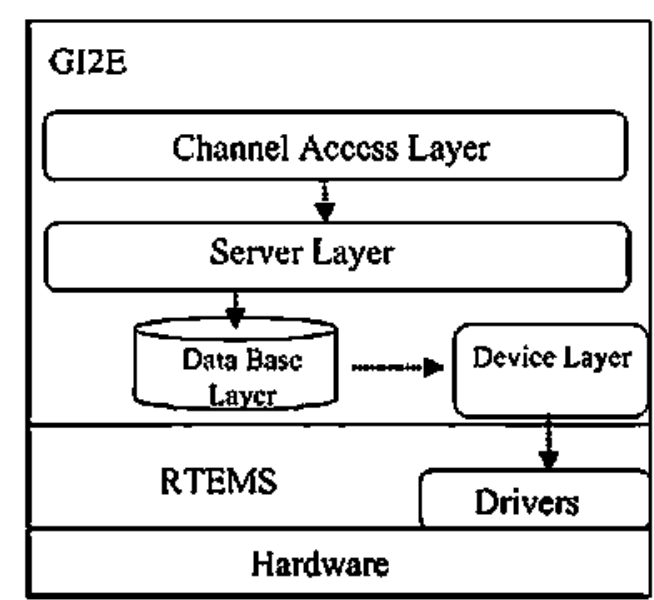

Fig. 3. GI2E's soltware architecture.

\subsection{GI2E's hardware architecture}

Fig. 2 shows the hardware architecture of the GI2E's module. The nucleus is a general purpose processor from Freescale's Coldfice family processors (MCF5234). Blocks in blue are in the processors' evaluation module M5234BCCKIT [3] and the rest have been implemented in a separate custom developed board to support the designed instrument.

The module works as a two port device, one with an Ethernet interface to connect to the EPICS system media, and another supporting several interface types to connect with the instrument that needs to be integrated. USB connections are also possible thanks to a RS232 to USB converter. The use of PHYTER's precision DP83640 permits to manage the IEEE1588 timestamps that travel through the network by hardware, obtaining a high degree of synchronization due to the elimination of latencies [4].

\subsection{GI2E's software architecture}

The module's software architecture follows the EPICS' five layer model. The first one is the Client-Server Layer. Clients running in the OPIs constitute the higher level layer of the software architecture. They are usually software applications that deal with the user interface, such as controlling panels, buttons, indicators, etc. The next one, Channel Access Layer, connects the clients with the servers hiding all the details of the TCP/IP implementation between them. This permits that both of them can run on different machines, with different hardware architectures and operating systems. The data interchange mechanism is optimized so data in the server

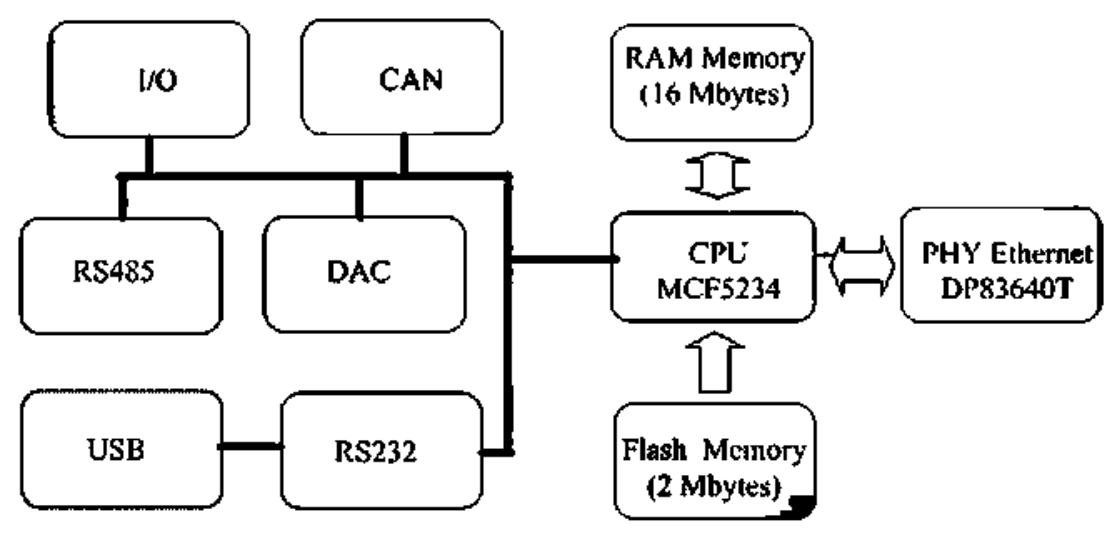

Fig. 2. Gl2E's hardware architecture. 
Examples of connection to EPICS systems

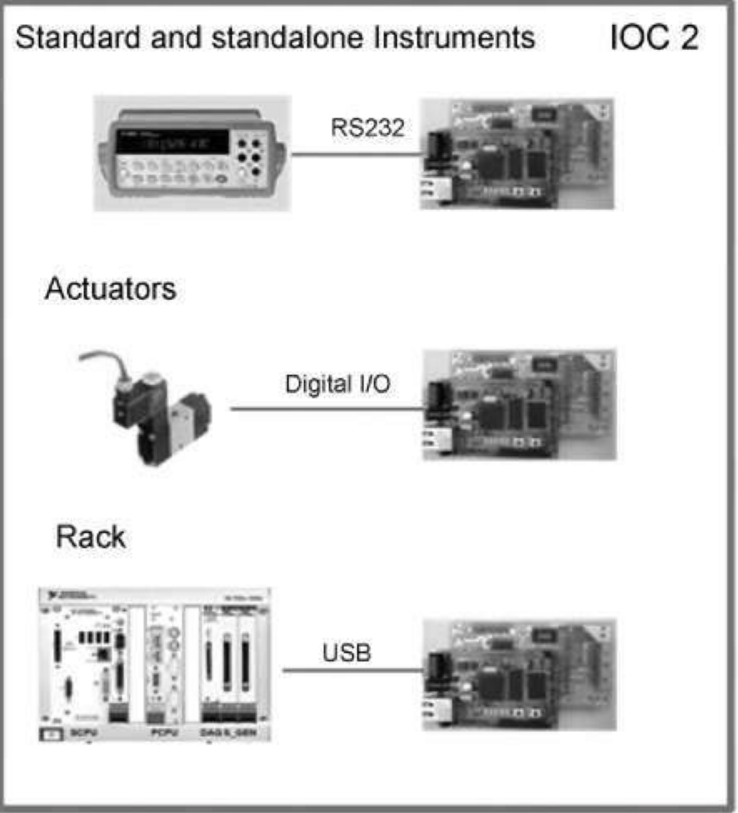

Fig. 4. Examples of connection to EPICS.

are only sent to the subscribed clients when there is a change in its value. The next one, Server Layer, isolates the clients from the database of the subsequent layers, whereas the fourth layer is the nucleus of the architecture. It defines a set of elements, named records, which defines the instrument behaviour by defining analog and digital input/outputs, waveforms, timers, etc. The fundamental entity of the database is the record structure, which is a register accessed by its name which contains a set of fields such as the range units, etc. Records can be accessed in three ways: using hardware interrupts, software events, or periodic scanning. Finally, the device layer is formed by specific hardware drivers from the hardware manufacturers. These drivers can also be used to communicate with external elements devices such as multimeters, actuators, etc.

Fig. 3 shows a schematic of the modules' software architecture. EPICS have been implemented over the RTEMS operating system. The use of RTEMS has permitted to implement the EPICS architecture with a very limited hardware platform as compared with traditional VME/VXI architectures [5]. The integration of EPICS is based in available portings from Coldfire boards that have been adapted for this architecture. A porting for the MCF5235 available in the processor's Freescale evaluation module was used. Some aspects had to be changed as the processor available in the board that was used was a different, but very similar one (MCF5234). Then a synchronization task based in PTP has to be developed. Additionally, a driver has been developed to access the characteristics of the PHYTER in the RTEMS environment.

Once the base EPICS software is installed the ASYN module is added to access RS232 devices. A database of the PV records has been developed based on this module The ASYN module has been configured to adapt it to the communication frames to ingrate. This permits to achieve the first goal, to be able to communicate different types of devices under the EPICS environment.

\section{GI2E applications}

GI2E lets include different instruments without EPICS capabilities and common communications interfaces in an EPICS architecture, basically three kinds of elements can be connected as is shown in Fig. 4.

The process to include an element in an EPICS system is:

- Select set of commands for instruments function.

- Implement them in command array.

- Modify EPICS database to handle the command array.

- Connect the equipment to GI2E using different interfaces: RS232, RS485, CAN, USB, $\mathrm{I} / \mathrm{O}$.

Fig. 5 shows a simplify schema of the system and the module software involved in the connection of instrument with RS232 functionality to an EPICS system [8].

Using the methodology above indicated the GI2E can be connected with ITMS architecture to improve synchronization features. ITMS is a high performance data acquisition platform based in PXI technology [6].

Typically a node of this distributed platform consists of a $6 \mathrm{U}$ chassis that includes an embedded CPU, named SCPU, which is used for the following tasks:

- Setup the data acquisition boards.

- Acquire data from the DAQ cards and distribute them among the CPUs installed in the system.

- Process some of this data.

The system will hold several data acquisition cards and CPUs to process the acquired data, named PCPUs increasing the processing capabilities of traditional PXI architectures.

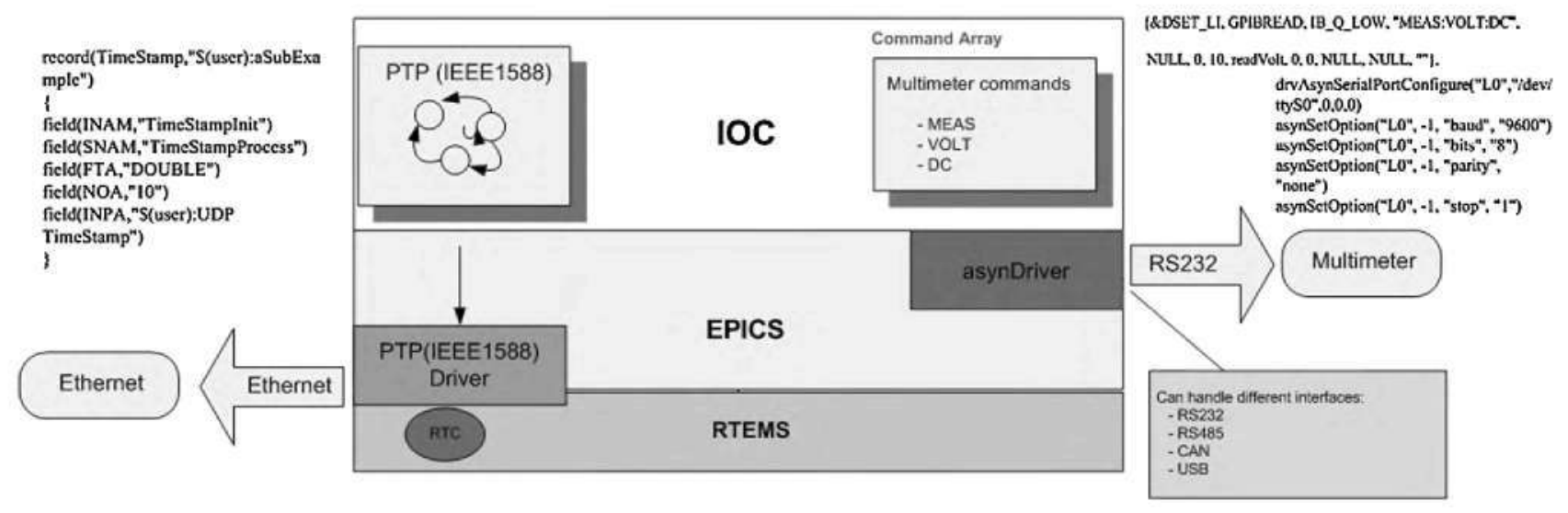

Fig. 5. Example of RS232 multimeter to EPICS connection. 


\section{DISTRIBUTED DATA ACQUISITION SYSTEM}

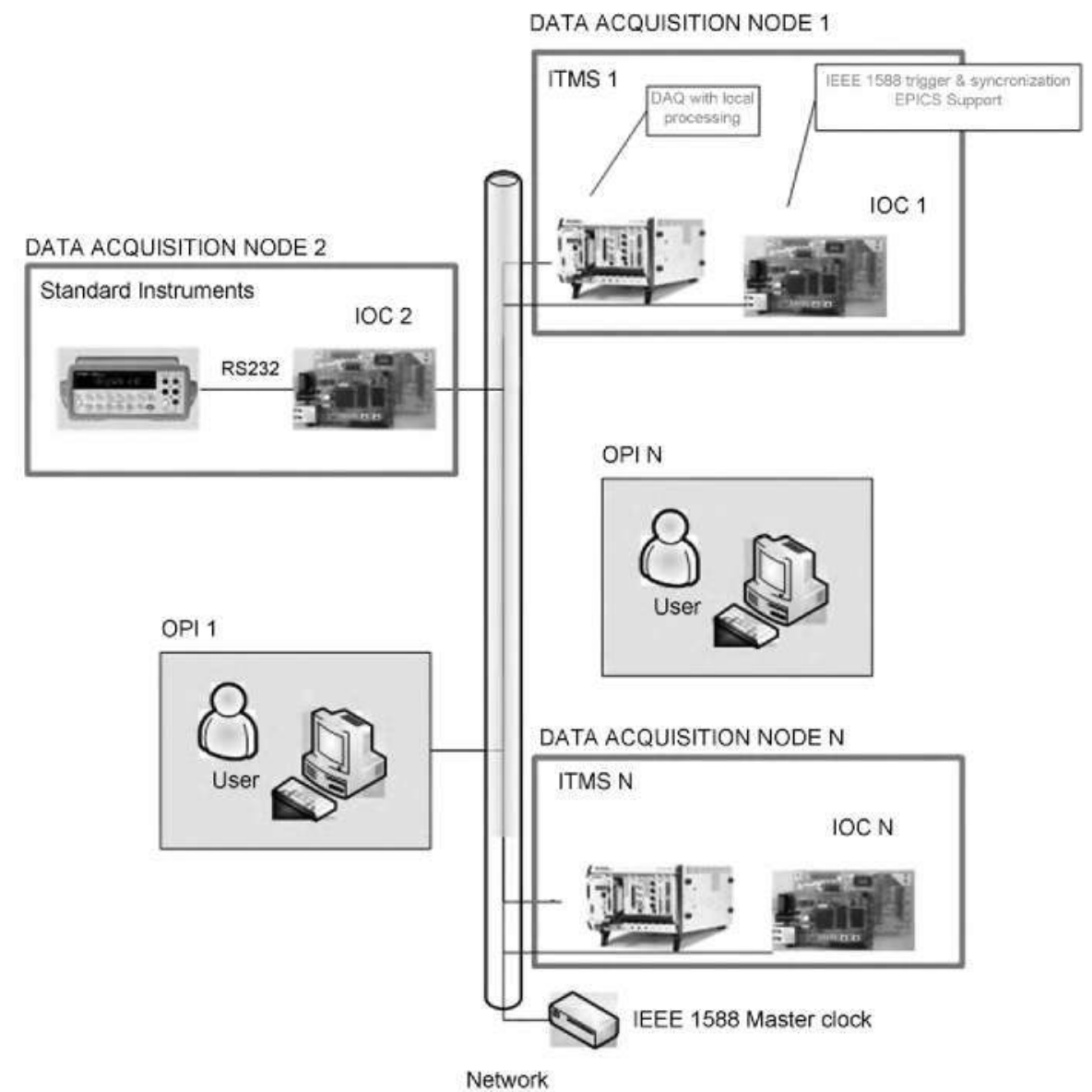

Fig. 6. TMS synchronization improvement using GI2E module,

ITMS has been used in several applications some of them in CIEMAT's TJ-II [7]. For example, an application with adaptive sampling capabilities based on hardware has been developed, in which determining the exact moment of the change in the sample rate was crucial. The PTP protocol proved to be very valuable to solve this problem [4]. The GI2E module has been used to improve the synchronization mechanisms of ITMS to interconnect several nodes as shown in Fig. 6.

\section{Experimental results}

In order to check the system performance, an adaptive sample rate solution has been implemented, the instrument setup experiment and measurements methodology is the same shown in [4]. After $24 \mathrm{~h}$ experiment, the trigger deviation between the master and the GI2E module was $68 \mathrm{~ns}$ with a standard deviation of $160 \mathrm{~ns}$ (probability of $95 \%$ ).

\section{Conclusions}

The inclusion of non standard or standalone instruments in EPICS systems it could be very interesting in order to solve specific problems that this kind of instrumentation has resolved. The module shows in this work is an easy method to connect different devices to EPICS systems. The mechanism is wide simple, the commands and/or actions to the devices have to be written in a command array file and modify the EPICS database according these requirements. This module in addition also let add a trigger for synchronizing distributed DAQ with very low uncertainty below $200 \mathrm{~ns}$. In particular the use of PTP-IEEE1588 standard, based in the use of Ethernet, is a powerful tool to implement distributed DAQ triggers [1,4]. New PHYTERs with hardware timestamping improve the precision of synchronization. The ITMS platform uses this module to implement high clock synchronization between the different chassis. As an example, an adaptive sample rate solution has been implemented.

\section{Acknowledgements}

This work is funded by the Spanish Ministry of Science and Technology under the Projects No. DPI200606624 and No. ENE2004-07335.

\section{References}

[1] International Standard, Precision clock synchronization protocol for networked measurement and cont rol system IEC61588, in: International Electrotechnical Commission, September, 2004.

[2] M.R. Kraimer, M. Clausen, W. Lupton, C Watson, Experience with EPICS in a wide variety of applications, in: Proceedings of Particle Accelerator Conference, Vol. 2, May, 1997, pp. 2049-2403.

[3] Freescale. http://www.freescale.com/webapp/sps/site/prod_summary.jsp? code=M5234BCCKIT.

[4] J.M. López, M. Ruiz, E. Barrera, G, de Arcas, J. Vega, Implementation of local area network extension for instrumentation standard trigger capabilities in advanced data acquisition platforms, Review of Scientific Instruments 79 (2008) 
[5] RTMES, littp:/www.ttems,org.

[6] E. Barrera, M. Ruiz, S. López, D. Machón, J. Vega, PXI-based architecture for real time data acqu isition and dist ributed dynamic data processing. IEEE TNS 53 (3) (2006) 923-926.

17] M. Ruiz, E. Barrera, S. López, D. Machón, J. Vega, E. Sánchez, Distributed real time data processing architecture for the TJ-II data acquisition system, Review of Scientific Instruments 75 (10) (2004) 4261-4264.

[8] P. Chevtsov, S. Schaffner, Information-control software for handling serial devices in an EPICS environment, in: Proceedings of the 8tli linternational
Conference on Accelerator and Large Experimental Physics Control Systems, November, 2001.

|9| D. Bashore, G. Oliaro, P. Roney. P. Siclita, K. Tindall, Conceptual design tor the NSTX central inst rumentation and contcol system, Fusion Engineeting 1 (October) (1997) 535-538.

|10| K.H. Kim, C.J. Ju, M,K, Kim, M.K. Patk, J.W. Choi, M.C. Kyum, et al., The KSTAR integrated control system based on EPICS, Fusion Engineering and Design 81 (2006) 1829-1833. 